Word count: 2, 562 including references \& table

\title{
SHARED MENTAL HEALTH CARE FOR A MARGINALISED COMMUNITY IN INNER-CITY CANADA
}

Stephen Kisely, MD, MSc

School of Medicine, Griffith University, Meadowbrook, Queensland, Australia, and Departments of Psychiatry and Community Health \& Epidemiology, Dalhousie University, Canada

Pamela Chisholm, RN, BScN

North End Health Community Health Centre and Capital District Health Authority (CDHA), Halifax, Nova Scotia,

Address for correspondence

School of Medicine, Room 2.15d, Building LO3, Logan Campus, Griffith University, University Drive, Meadowbrook Qld 4131, AUSTRALIA

Phone Number: 61 (0)7 33821320 Fax Number: 61 (0)7 33821338 Email:

s.kisely@griffith.edu.au 


\section{ABSTRACT}

Objectives: We describe the experience and evaluation of a shared care project targeted at marginalised individuals living in the North End of Halifax, Nova Scotia. This population has high rates of psychiatric disorder often co-morbid with chronic medical conditions, and have difficulty in obtaining the help they need. This primary care liaison service covers all ages and includes outreach to emergency shelters, transitional housing and drop-in centres.

Collaborative care improved access, satisfaction and outcomes for marginalised individuals in urban settings. Primary care providers with access to the service reported greater comfort in dealing with mental health problems, and satisfaction with collaborative care, as well as mental health services in general. Results were significantly better than those of control practices when such data were available. The median wait time was 6 days in comparison with 39.5 for the comparison site.

Conclusions: This model can complement other initiatives to improve the health of marginalised populations, and may be relevant to Australia.

Key Words: primary care, collaborative care, shared care, psychiatric disorder; marginalised populations. 


\section{Introduction}

Up to $50 \%$ of patients seen in primary care have mental health problems, the severity and duration of which are often similar to those of individuals seen in the specialised sector $[1,2]$. Shared or collaborative care is not restricted to mental health but can apply to any "positive interaction of two or more health professionals, who bring their unique skills and knowledge, to assist patients/clients and families with their health decisions” [3].

Although initial models emphasised collaboration between general practitioners (GPs), psychiatrists and nurses, collaborative care has expanded to involve patients, psychologists, social workers, occupational therapists, pharmacists and other providers [4]. Other developments have seen collaborative care aimed at specific groups such as children, adolescents, the over-65s and marginalised individuals who find access to services difficult. Collaborative care can include attachment or shared-base models, where mental health professionals see patients in primary care, and consultation-liaison services where GPs and others are provided with education, advice, support and second opinions in their assessment and management of mental health problems [5].

This paper describes our experience of a primary care liaison service with community outreach targeted at marginalised individuals living in Halifax (population=372, 679), the provincial capital of Nova Scotia, Canada. The urban marginalised include individuals who are homeless, living with addiction, living with disabilities, street youth, sole support parents, Aboriginal Peoples, mentally ill persons, gay/lesbian/bisexual/ transgendered individuals, and racial minorities (including immigrants and refugees), who live in an urban setting and share common determinants related to social exclusion and poverty [6].

Under the Canada Health Act, all Canadian residents are entitled to in-patient or out-patient care that is free at the point of delivery. Patients receive treatment at 
publicly-funded facilities or are seen by private psychiatrists or general practitioners in the community, who then bill the provincial health plan.

\section{The locality}

The programme serves the North End, a socially deprived inner city area of Halifax. Median household income is in the lowest $10 \%$ of the range in Halifax [7]. The area houses a large number of emergency shelters, transitional housing and drop-in centres for marginalised groups (Table 1).

The service forms part of the collaborative care programme of in the Capital District Health Authority (CDHA) of Nova Scotia. CDHA includes the metropolitan area of Halifax, as well as surrounding rural areas and covers approximately $40 \%$ of Nova Scotia’s population. This wider programme covers ten sites throughout CDHA [8]. The services operates out of the North End Community Health Centre (NECHC). The centre was founded in 1971 by local residents in response to a need for health care services in the North End [9]. NECHC is primarily funded by the Provincial Department of Health managed through the Capital District Health Authority [9]. Health care services are provided by five nurses/nurse practitioners, five GPs, one addiction counsellor, a dietician and social worker [9].

\section{The need}

Mental illness and homelessness are interrelated in that people who are homeless have high rates of psychiatric morbidity, while individuals with severe and chronic mental illness are more likely to become homeless [10]. The most prominent mental disorders in homeless adults are depression, affective disorders, substance abuse, psychotic disorders, schizophrenia, and personality disorders [11,12]. Approximately 75\% of a study population in Alberta reported 
some psychiatric symptoms, while almost one-third reported significant symptoms [13]. Over $60 \%$ report problems with alcohol consumption, while illicit or prescription drug misuse estimates range from 20 to $30 \%$ [11].

Data for Halifax reveal a similar picture. A study based on qualitative interviews with homeless individuals, and a one-day snapshot survey, reported that nearly two thirds had some health condition; 33\% reporting addiction; 20\% mental illness; 15\% a medical condition; and $4 \%$ disability [14].

In addition, focus groups with primary care practitioners, community agency staff and consumers in the Halifax area in early 1998 revealed considerable dissatisfaction with local mental health care provision, including difficulties with access, referral and communication among health care providers [15].

\section{The service}

The service began in 1997 at the North End Community Health Centre (NECHC) to serve the needs of marginalised populations, or those who are less likely to gain access to mental health services. The project was initially funded through Health Canada’s Health Transition Fund before being assumed by the local health authority. It offers child and adolescent, as well as adult, mental health services. The service initially consisted of one part-time adult psychiatrist and one full-time mental health nurse to provide clinical support in direct care, consultation, liaison and education. Over time, this has expanded to include social workers, other psychiatrists and family practices, with ebbs and flows in staff numbers.

Consultation-liaison to primary care is complemented by outreach to emergency shelters, transitional housing and drop-in centres including needle exchange and methadone maintenance programmes (Table 1). This increases access, improves continuity and relevancy of care, and avoids duplication of services. Regular visits by the mental health worker allow a 
comprehensive view of individuals’ physical and mental health, housing, finances, social ties, and employment through direct daily observation. In these settings, care team members may observe levels of functioning, and gain insight into areas of concern, not readily reported or detected in a community health centre [6].

The service is aimed at individuals who have difficulty in obtaining help through the traditional system, or clients of front-line social agencies in frequent crises without appropriate supports. Other targets are primary care and front-line agency staff who are the initial contact for individuals in crisis, but who lack the time, training and support necessary to help.

All team members are salaried to allow for compensation when undertaking non-billable services such as care co-ordination and, educational sessions. The full-time mental health worker acts as the liaison between the individual presenting with problems, agency staff, the shared care team and all other necessary community resources. After referral from agency staff, or another advocate (including self-referral), the mental health worker decides if a referral to the GP is appropriate and when to involve other shared care team members including the psychiatrist. The primary and shared mental health teams also discuss problems at weekly care co-ordination meetings. These include the psychiatrist and other mental health workers, nurses, GPs, dietician and social worker. There are also educational sessions at sixmonthly retreats for primary care teams and mental health professionals at all the collaborative care sites in the CDHA programme.

\section{The effect}

There have been two evaluations of the service. The first was a mixed-methods survey two years after implementation [15]. Data collection lasted over 12 months and included the NECHC, as well as two other sites offering collaborative care: an urban family medicine 
centre and a rural family practice. A pretest-posttest design was used, and where possible the three intervention sites were compared to a control GP clinic without access to the service. All settings were a convenience sample. Outcomes studied included waiting times to being seen my a mental health worker, number of subsequent referral to psychiatrists as opposed to other disciplines and primary care staff satisfaction with the service. The NECHC and control clinic were similar in terms of staffing (13 and 16 full time equivalents respectively). 243 patients were recruited at NECHC but only 29 at the control clinic. They were similar in terms of age (approximately 30 years old) and female gender (48\% vs. $60 \%)\left(\chi^{2}=1.14, \mathrm{df}=1, \mathrm{p}=0.38\right)$ [15]. NECHC had a higher proportion of homeless patients [15]

Access was partly assessed by the median wait time defined as the number of days between referral from a primary care provider to being seen by a mental health professional. The median wait time at the NECHC was 6 days in comparison with 39.5 for the comparison site. There were statistically significant improvements in general (44 to 56) and mental health (40 to 53) as measured by the DUKE Health Questionnaire, not mirrored in the comparison site (37 and 39 to 45 respectively) [15]. However, this may be due to the fact that only 10 subjects agreed to complete the questionnaire in the control practice. Patient satisfaction on the Visit Specific Questionnaire was high, with between 71\% and 78\% being satisfied to extremely satisfied with the time to being seen, being seen in the community centre and their visit overall [15].

In terms of the qualitative research, general practitioners also expressed satisfaction with the service and felt that collaboration has increased their knowledge and confidence in diagnosing and treating individuals with mental health problems [15]. There were no data from the control site on patient or general practitioner satisfaction with the service. 
This limitation was partly addressed by a subsequent survey of general practitioners $(n=101)$ in Capital District Health Authority [8]. This revealed that GPs who had access to collaborative care reported significantly greater knowledge in the areas of psychosis, and childhood behavioural problems [8]. General practitioners in contact with collaborative care were also significantly more satisfied with mental health services in general, over and above shared care [8]. All these results remain significant even after controlling for sex, level of interest and years of practice [8]. However it was not possible to identify how many of the participating physicians came from the NECHC.

\section{Limitations}

Limitations of the model include the generalisability to other settings such as 'fee-for-service' clinics. In the absence of provincial funding, the local health authority has remunerated general practitioners for activities such as education or case conferences not covered by provincial billings. Another difficulty has been adequate funding and personnel, especially to meet the needs of patients under 19 years old. Finally, the model may be more applicable to urban settings with a high concentration of shelters, transitional housing schemes and drop-in centres where contact can be made. It may be less applicable to rural areas.

In terms of the evaluation, limitations include the absence of sufficient control data for many of the measures. Participation rates for some measures were low and neither of the evaluations was specifically of the service for marginalised populations. Where control data are available for comparison, it has not always been possible to separate out the effect of this particular service from other collaborative care programmes in the Halifax area. Other limitations include the absence of data on GP work practices, knowledge or interests concerning mental 
health care before the introduction of collaborative care, and the consequent reliance on retrospective information. Self-report data are subject to information bias.

\section{Conclusions}

Collaborative care can improve access, satisfaction and outcomes for marginalised individuals in urban settings although further research is required given the limitations of the existing data. The results may be relevant to Australia. Primary care providers with access to the service reported greater comfort in dealing with mental health problems, and satisfaction with collaborative care, as well as mental health services in general. Results were significantly better than those of control practices when such data were available. This model can complement other initiatives to improve the health of marginalised populations such as movement away from large temporary shelters and institutions, in favour of longer-term supportive housing where tenants have enhanced access to health and community services with or without residential supervision. 


\section{REFERENCES}

1 Kates N, Fugere C, Farrar S. Family Physician Satisfaction with Mental Health Services, Findings from a Community Survey. CPA Bulletin 2004; 36:10-14.

2 Lesage A, Goering P, Lin E. Family physicians and the mental health system, a report from the mental health supplement to the Ontario Health Survey. Can Fam Physician 1997; 43:25-6.

3 Enhancing Interdisciplinary Collaboration in Primary Health Care. The Principles and Framework for Interdisciplinary Collaboration in Primary Health Care, 2005 Available from URL: http,//www.eicp-acis.ca/en/ principles/sept/EICP-Principles\%20and\%20 Framework\%20Sept.pdf

4 Craven MA, Bland R. Better practices in collaborative mental health care, An analysis of the evidence base. Can J Psychiatry 2006; 51:7S-72S.

5. The Royal Australian College of General Practitioners \& The Royal Australian \& New Zealand College of Psychiatrists. Primary Care Psychiatry: The Last Frontier. A report of the Joint Consultative Committee in Psychiatry. A National Mental Health Strategy Project, 1997.

6 Canadian Collaborative Mental Health Initiative (CCMHI). Establishing collaborative initiatives between mental health and primary care services for urban marginalized populations. Ottawa: Canadian Collaborative Mental Health Initiative, 2006. Available from URL: http://www.ccmhi.ca/en/ products/toolkits/documents/EN_CompanionToolkitforUrbanMarginalized.pdf

7 Statistics Canada. Spatial Structure of census tracts by household income. Ottawa, Ontario: Statistics Canada, Geography Division, 2001. 
8 Kisely S, Duerden D, Shaddick S, Jayabarathan A. Increased Collaboration Between Primary Care and Psychiatric Services. Can Fam Physician 2006; 52:876-877. Available from URL: http://www.cfpc.ca/cfp/2006/Jul/vol52-jul-researchkisely.asp?stype=advanced $\&$

9. North End Community Health Centre. About the Centre. Available from URL: http://www.nechc.com/

10. Hwang SW. Homelessness and health. CMAJ 2001; 164:229-3.

11 Acorn S. Mental and physical health of homeless persons who use emergency shelters in Vancouver. Hosp Community Psychiatry 1993; 44:854-7.

12 Reilly JJ, Herrman HE, Clarke DM, Neil CC, McNamara CL . Psychiatric disorders in and service use by young homeless people. MJA 1994; 16:429-32.

13 Stuart HI, Arboleda-Flórez J. Homeless shelter users in the post-deinstitutionalization era. Can J Psychiatry 2000; 45:55-62.

14 Halifax Regional Municipality. Homelessness in HRM, A Portrait of Streets and Shelters, Vol. II. Planning and Development Services, Halifax, NS, 2005.

15 McLean L, Rowan M, LeBlanc J. Improving identification, early intervention and outcomes for people with mental illnesses in the community, a model for primary care services integration in the area of mental health. Report to Health Transitions Fund. Ottawa Ontario: Health Canada, 2000. Available from URL: http://www2.itssti.hcsc.gc.ca/hpb/hcpd/pchcd/projectc.nsf/ExecSum/NS421/\$File/NS421.pdf 
Table 1: Emergency shelters, transitional housing and drop-in centres in Halifax

\begin{tabular}{|l|l|}
\hline Emergency Shelters & emergency for youth over 16, women and children \\
\hline Adsum House & transitional housing for women \\
\hline Adsum Centre & emergency for women and children \\
\hline Barry House & $\begin{array}{l}\text { transitional housing for women and children escaping } \\
\text { abuse }\end{array}$ \\
\hline Bryony House/Veith House & emergency for men \\
\hline Metro Turning Point & emergency for youth \\
\hline Phoenix Youth Shelter & emergency for single men and addictions program \\
\hline Salvation Army & supported transitional housing for women and children \\
\hline Transitional Housing & residential program for men with addictions \\
\hline Alice Housing & residential program for men with addictions \\
\hline Al-Care Place & residential program for women with addictions \\
\hline Freedom Foundation & Supported housing for women \\
\hline Marguerite Centre & \\
\hline YWCA of Halifax & breakfast program \\
\hline Drop-in centres & drop in centre for homeless youth \\
\hline Brunswick St. United Church & support centre for homeless and at risk individuals \\
\hline Phoenix Centre for Youth & drop in centre for homeless youth at risk \\
\hline $\begin{array}{l}\text { Metro Non-profit Housing } \\
\text { Support Centre }\end{array}$ & soup kitchen \\
\hline ARK & needle exchange program and outreach centre \\
\hline Feeding Others of Dartmouthe program \\
\hline Mainline & \\
\hline Direction 180 & methadone \\
\hline
\end{tabular}

